\title{
Déficit estatural em crianças em idade escolar e em menores de cinco anos: uma análise comparativa
}

\author{
Short stature in school aged and under-five year \\ old children: a comparative analysis
}

Glória Elizabeth Carneiro LAURENTINO'

Ilma Kruze Grande de ARRUDA ${ }^{2}$

Maria Cristina Falcão RAPOSO 3

Malaquias BATISTA FILHO ${ }^{4}$

\section{R E S U M O}

\section{Objetivo}

Investigar se a ocorrência de déficit estatural em crianças em idade escolar seria semelhante à encontrada em menores de cinco anos, em termos de prevalência e de alguns fatores de risco.

\section{Métodos}

A amostra foi constituída de 894 crianças em idade escolar (entre seis e doze anos) e de 2078 crianças menores de cinco anos. Adotou-se o teste $\chi^{2}$ de associação de Pearson para testar, em cada um dos grupos estudados, a associação entre o déficit estatural e os possíveis fatores de risco. Utilizou-se o teste de igualdade de proporções para verificar as diferenças das prevalências de déficit estatural em cada grupo de idade e ajustou-se um modelo multivariado explicativo do déficit estatural em menores de cinco anos.

\section{Resultados}

Considerando o total da amostra, a proporção de déficit estatural entre crianças em idade escolar $(16,9 \%)$ foi significativamente maior, quando se comparou com o valor percentual $(12,1 \%)$ obtido entre os menores de cinco anos. O modelo multivariado indicou que o déficit estatural da criança em idade escolar, a renda per capita, a escolaridade e a idade materna foram os fatores que melhor explicaram o déficit estatural das crianças menores de cinco anos.

\footnotetext{
1 Departamento de Fisioterapia, Centro de Ciências da Saúde, Universidade Federal de Pernambuco. Av. dos Reitores, s/n, Cidade Universitária, Campus Universitário, 50670-420, Recife, PE, Brasil. Correspondência para/Correspondence to: G.E.C. LAURENTINO. E-mail: <gloriaecl@ufpe.br>

2 Departamento de Nutrição, Centro de Ciências da Saúde, Universidade Federal de Pernambuco. Recife, PE, Brasil.

3 Departamento de Estatística, Centro de Ciências Exatas e da Natureza, Universidade Federal de Pernambuco. Recife, PE, Brasil.

${ }^{4}$ Instituto Materno Infantil de Pernambuco. Recife, PE, Brasil.
} 


\section{Conclusão}

Nas áreas urbanas do Estado de Pernambuco, o indicador altura/idade pode ser indicativo de colinearidade entre crianças em idade escolar e menores de cinco anos. A ocorrência de déficit estatural na criança em idade escolar foi o principal fator preditivo do déficit estatural no irmão menor de cinco anos.

Termos de indexação: criança; déficit estatural; nanismo nutricional; saúde escolar.

\section{A B S T R A C T}

\section{Objective}

To investigate whether the occurrence of short stature in school-aged children would be the same as that found in under-five year olds in terms of prevalence and some risk factors.

\section{Methods}

The sample consisted of 894 school-aged children (from 6 to 12 year-olds) and of 2078 under-five year olds. Pearson's $\chi^{2}$ test of association was employed to verify the association between short stature and possible risk factors in each of the groups investigated. The equality of proportions test was used to verify the differences in prevalence of short stature in each age group, and an explanatory multivariate model of the short stature in the under-five year olds was adjusted.

\section{Results}

Taking into account the entire sample, the proportion of short stature amongst school-aged children (16.9\%) was significantly greater than amongst under-five year olds (12.1\%). The multivariate model showed that short stature in school-aged children, the per capita income and the mother's educational level and age, were the factors that best explained short stature in the under-five year olds.

\section{Conclusion}

In urban areas of the State of Pernambuco, the height/age indicator may be indicative of co-linearity between school-aged children and under-five year olds. The occurrence of short stature in school-aged children was the main predictive factor of short stature in under-five year old siblings.

Indexing terms: child; short stature; nutritional dwarfism; school health.

\section{N T R O D U Ç Ã O}

O crescimento somático, sobretudo em relação à estatura, expressa, em âmbito individual e, por extensão, em escala populacional, a interação de fatores positivos e negativos de diversas naturezas e intensidades, modulando os processos geneticamente programados. $\mathrm{Na}$ definição alegórica de Tanner ${ }^{1}$, estatura é status, uma vez que o crescimento longitudinal se correlaciona com a posição social, sendo favorecido pelas boas condições de vida e dificultado nas situações de adversidades socioeconômicas. O resultado final é a nítida diferenciação de estatura entre povos de países subdesenvolvidos e nações avançadas, ou entre ricos e pobres de uma mesma sociedade, num mesmo tempo e com a mesma formação racial ${ }^{2,3}$.
No ser humano, o crescimento ocorre durante um período de vida relativamente longo, passando por diversas fases que podem transcorrer em diferentes ecossistemas físicos, biológicos e sociais. Nesse trânsito, estabelecem-se variadas situações de vulnerabilidade e risco, entre as quais as condições de saúde e nutrição exercem papéis preponderantes, de forma que a estatura, em seu estágio final e em seus processos intermediários, representa a história cumulativa do jogo entre fatores favoráveis e de handicaps que possibilitam a afirmação ou, ao contrário, a frustração do potencial biologicamente estabelecido pela "bagagem" genética ${ }^{4,5}$.

Assim, nos primeiros meses e anos de vida, período de acelerado crescimento e elevadas demandas nutricionais, além de se expor a diferentes adaptações ambientais e mudanças na 
alimentação, desde o aleitamento materno exclusivo até a diversificação dietética da rotina familiar, a criança é mais susceptível às intercorrências patológicas, especialmente às infecções. Essas particularidades, associadas à circunstância de que o crescimento praticamente se estabiliza até os primeiros 24 meses de vida, tornam essa fase crucial para o processo de crescimento infantil ${ }^{6,7}$. Dessa forma, sugere-se que, em nível populacional, os déficits de altura, observados no final da idade pré-escolar (menores de cinco anos), representam, com boa aproximação, os retardos estaturais que serão encontrados na idade adulta ${ }^{7,8}$.

Na idade escolar, quando já foram superados os riscos mais marcantes que comprometem o estado nutricional, a baixa estatura expressa muito mais os déficits de crescimento ocorridos no passado do que a situação presente ${ }^{4,7,9}$. Assim, o escolar representa a história e o resultado da cinética nutricional desde a vida intra-uterina até a seqüência lactente/ pré-escolar e o próprio escolar ${ }^{10}$, tornando esse grupo privilegiado para avaliação nutricional da população infanto-juvenil ${ }^{11}$.

Com essas características, a medida da altura de escolares vem sendo sugerida como um excelente indicador para caracterizar e acompanhar o estado de saúde e nutrição da população na qual se inserem esses escolares. O primeiro autor a fazer essa recomendação parece ter sido Bengoa. Sua proposta, já na década de 70, baseava-se na maior vulnerabilidade da população infantil aos agravos ambientais e na constatação de que a relação altura/idade, na idade escolar, resume muito bem as conseqüências dos eventos sociais, econômicos e biológicos ocorridos com as crianças desde sua concepção ${ }^{12}$.

Essa recomendação, apoiada por organismos internacionais e pesquisadores da área ${ }^{13-17}$, vem sendo testada em alguns países da América Latina, como Costa Rica ${ }^{13-16}$, Panamá13,16 e Nicarágua ${ }^{13-15}$, onde levantamentos censitários da estatura de escolares são utilizados como suporte para definição de políticas de alimentação e nutrição. No Brasil, além da experiência de Mondini et al. ${ }^{17}$, em São Paulo, foram realizados alguns estudos em estados do Nordeste brasileiro, como Ceará18, ${ }^{18}$ araíba ${ }^{19}$ e Piauí20, mediante censos de escolares ingressantes na primeira série do primeiro grau, para verificação da prevalência de déficit estatural como um indicador epidemiológico do estado de nutrição energéticoprotéica, configurando as áreas de risco e discriminando, comparativamente, a magnitude do problema.

Sem descartar a pertinência e a importância dos dados de censos de altura, torna-se necessário realizar, periodicamente, inquéritos domiciliares de amostras representativas da população que incluam um conjunto diversificado de variáveis para entender, analiticamente, os fatores implicados na rede causal dos problemas nutricionais e sua distribuição geográfica, populacional e temporal ${ }^{9}$.

Dessa forma, partindo-se do pressuposto de que a prevalência do déficit estatural encontrado em crianças em idade escolar seria semelhante aos valores de ocorrência em crianças menores de cinco anos, este estudo tem por objetivo investigar até que ponto tal hipótese poderia ser aceita para retratar o quadro da desnutrição infantil no Estado de Pernambuco. Nessa perspectiva, propõe-se comparar a prevalência do déficit de altura/idade $(A / I)$ em crianças em idade escolar e menores de cinco anos, bem como os possíveis fatores de risco (biológicos e socioeconômicos) nos dois grupos e ajustar um modelo multivariado, admitindo, hipoteticamente, a possibilidade de explicar o retardo estatural em menores de cinco anos a partir do comprometimento estatural de seus irmãos em idade escolar.

\section{M É T O D O S}

Este estudo, do tipo transversal, foi desenvolvido tendo como fonte de dados o arquivo da II Pesquisa Estadual de Saúde e Nutrição do Estado de Pernambuco - $1997^{21}$ e como referência 
analítica a dissertação "Prevalência da Desnutrição em Crianças Menores de 5 Anos, do Estado de Pernambuco e sua relação com alguns fatores de risco"- 199722, também originária do mesmo banco de dados.

Na pesquisa ${ }^{21}$ fonte dos dados deste artigo, foi considerada como unidade de estudo a família com criança menor de cinco anos e, a partir desse critério, foram estimadas subamostras para representar estratos e grupos populacionais em função de objetivos específicos. Os dados foram registrados em formulário próprio, preenchido mediante entrevistas domiciliares no período de fevereiro a maio de 1997. As medidas antropométricas foram realizadas em duas tomadas e colhidas no momento da entrevista. A altura das crianças até dois anos foi obtida em decúbito dorsal e aquelas entre dois e cinco anos, em posição ereta, descalças, com um infantômetro confeccionado em barra de madeira, com amplitude de $100 \mathrm{~cm}$ e subdivisões de $0,1 \mathrm{~cm}$. A altura das crianças em idade escolar foi determinada com fita métrica de $200 \mathrm{~cm}$ marca Stanley - milimetrada, com precisão de até $1 \mathrm{~mm}$ em toda sua extensão. O detalhamento do método adotado no estudo anteriormente referido encontra-se disponível nas publicações citadas.

A amostra do tipo probabilística (aleatória estratificada) foi dimensionada para representar as crianças menores de cinco anos residentes em três áreas geográficas (Região Metropolitana do Recife (RMR), Interior urbano (IU) e Interior rural (IR). Entretanto, é possível considerar que as crianças em idade escolar - residentes em habitações onde foram encontrados os menores de cinco anos, quase sempre seus irmãos - possam representar o universo de crianças nessa idade para o Estado de Pernambuco. A amostra foi constituída de 1013 crianças em idade escolar (entre 6 e 12 anos) - das quais $119(11,7 \%)$ não foram incluídas por falta de informações antropométricas, totalizando, assim, 894 crianças em idade escolar - e de 2078 crianças menores de cinco anos. Para ambos os grupos de idade foi observada ausência de informação relativa a algumas variáveis e, por essa razão, nos resultados apresentados, os percentuais não foram calculados considerando a totalidade da amostra.

$\mathrm{Na}$ análise inicial foi considerado como variável dependente o déficit estatural segundo o indicador altura/idade (A/I), tomando-se como ponto de corte o limite referente a -2 escore- $Z$ $(Z<-2)$, tendo como referência o padrão do National Center of Health Statistics (NCHS), recomendado pela Organização Mundial de Saúde para uso internacional ${ }^{23}$. Assim, foram consideradas crianças com déficit estatural (baixas para idade) aquelas classificadas abaixo de -2 escore-Z $(Z<-2)$, e com altura adequada para a idade (eutróficas) aquelas com valores maiores ou iguais a $-2(Z \geq-2)$.

Para análise bivariada foram consideradas, além do gênero das crianças, algumas variáveis socioeconômicas da família, como abastecimento de água, por meio da rede geral (com canalização interna) e de outras formas (poço ou nascente, chafariz, rede geral sem canalização interna e outras formas); tratamento da água de beber: tratada (fervida, filtrada, mineral ou clorada) e sem tratamento (coada, sem tratamento e outras formas); esgotamento sanitário: sanitário ligado à rede geral, sanitário ligado à fossa com tampa e outras formas (incluindo sanitário ligado à fossa rudimentar); destino do lixo: coletado e outras formas (incluindo a colocação em terreno baldio e outras formas); posse do bem geladeira; renda familiar mensal e renda per capita. Para a renda familiar mensal foram consideradas as distintas fontes de rendimento dos diversos membros da família, derivadas do trabalho assalariado e de outras origens. O total obtido foi transformado, para efeito de análise comparativa, em frações do salário mínimo (SM), adotando-se as categorias: abaixo de $1 \mathrm{SM}(<1 \mathrm{SM})$, de 1 a menos de 2 SM $(1 \vdash 2)$ e maior ou igual a 2 SM $(\geq 2 S M)$.

A renda per capita foi calculada dividindo-se a renda familiar total, em SM, pelo número de pessoas do domicílio, adotando-se a seguinte estratificação: abaixo de $1 / 4$ do SM $(<0,25)$, de $1 / 4$ a menos de meio SM $(0,25 \vdash 0,50)$ e maior ou 
igual a 0,50 SM $(\geq 0,50)$. Na ocasião da pesquisa, um salário mínimo correspondia a $\mathrm{R} \$ 120,00$ (equivalente a US\$113.42).

Para a análise estatística dos resultados foi empregado o teste Qui-quadrado $\left(\chi^{2}\right)$ de associação de Pearson e, quando pertinente, a correção de Yates para testar a associação entre o estado nutricional e os fatores de risco em cada grupo. No caso de variáveis com mais de duas categorias, sendo essas ordinais, foi utilizado o teste $\left(\chi^{2}\right)$ para tendência linear.

Para análise das diferenças entre as proporções de déficit estatural em cada grupo de idade (segundo fatores de risco), utilizou-se o teste de igualdade de proporções (teste com estatística Z), no qual a hipótese $\mathrm{H}_{0}$ admite que as prevalências de déficit estatural entre os grupos de idade (crianças menores de cinco anos e em idade escolar) seriam iguais. Para esse teste tem-se como valor crítico $Z=1,96$, considerando um intervalo de $95 \%$ de confiança.

Posteriormente, fez-se o sorteio de uma subamostra de crianças em idade escolar (um em cada residência), sorteando-se, também, em cada uma dessas residências, uma criança menor de cinco anos, resultando, assim, na subamostra, a razão de 1:1 para crianças em idade escolar e menores de cinco anos.

Para a análise multivariada, além das variáveis socioeconômicas da família utilizadas na análise bivariada, descrita anteriormente, foram consideradas também variáveis biológicas das crianças (gênero e idade) e variáveis maternas, como idade e escolaridade. Para as crianças, a idade foi categorizada em duas faixas etárias, de 6 a menos de 10 anos $(6 \vdash 10)$ e maior ou igual a $10(\geq 10)$ para aquelas em idade escolar, e de zero a menos de 2 anos $(0 \vdash 2)$ e maior ou igual a $2(\geq 2)$ para os menores de 5 anos. Com relação às variáveis maternas, foi assumido que a mãe da criança em idade escolar da subamostra sorteada seria a mesma do menor de 5 anos; segundo a Pesquisa Nacional por Amostra de Domicílio de 2002 (PNAD) ${ }^{24}$, essa chance seria de $91,5 \%$, visto que esse é o percentual de domicílios onde reside apenas uma família.
Nessas condições, foi construído um novo banco de dados com as informações dos irmãos (em idade escolar e menores de 5 anos) e suas mães. Como critério de exclusão para os 8,5\% de possíveis dúvidas no pareamento mãe/filho, foi calculada a diferença de idade entre a criança em idade escolar e sua mãe, admitindo-se um valor mínimo de 14 anos, ou seja, 14 anos seria a idade mínima aceitável para que a mulher pudesse ter sido mãe. Assim, o banco final de dados resultou em 558 registros de irmãos. As variáveis maternas (idade e escolaridade) foram incluídas no modelo multivariado como variáveis contínuas.

A partir do banco de dados dos irmãos foi elaborada, inicialmente, uma análise bivariada, tal como já descrita, para testar a associação entre o déficit estatural da criança menor de cinco anos e os possíveis fatores de risco, além de calcular o valor da Odds ratio $(O R)$ para ocorrência de déficit estatural na criança menor de cinco anos.

A análise multivariada foi realizada ajustando-se um modelo de regressão logística, com variável dependente binária $(Y=1$, representando a criança menor de cinco anos com déficit estatural e $Y=0$, a criança menor de cinco anos sem déficit estatural) e como variáveis explicativas, além da condição nutricional de seu irmão em idade escolar, variáveis socioeconômicas da família, informações biológicas das crianças e variáveis maternas, usando como critério de seleção o método stepwise, considerando-se sempre um nível crítico de 95\% de confiança na elaboração da totalidade dos cálculos $(p \leq 0,05)$. Com esse modelo pretende-se prever a condição estatural de uma criança menor de cinco anos a partir da estatura do seu irmão em idade escolar e mais alguns fatores de risco associados. Definindo que a probabilidade de $Y=1$ representa $p$, então o modelo linear ajustado que considera a transformação logística, no qual o Logit $(Y)=\log (p /(1-p))$, é dado por: Logit $(Y)=\alpha+\beta X$, sendo $\alpha$ e $\beta=\left(\beta_{1}, \beta_{2}, \beta_{3}, \ldots, \beta_{k}\right)$ os parâmetros a serem estimados e $X=\left(X_{1}, X_{2}, X_{3}, \ldots, X_{k}\right)$ as variáveis explicativas. 
A partir dos valores dos parâmetros, foi então calculado, além do odds ratio, o valor estimado da probabilidade de uma criança menor de cinco anos ter comprometimento de estatura em função da estatura de seu irmão em idade escolar e de outras variáveis estatisticamente significativas, da seguinte forma:

$$
P(Y=1)=p=\frac{e^{\alpha+\beta x}}{1+e^{\alpha+\beta x}}
$$

As plataformas computacionais utilizadas foram os softwares Epi Info versão 6.02 e SPSS, versão 10.0 para Windows.

\section{RES U LTA DOS}

No Estado de Pernambuco, a situação do domicílio comportou-se como um discriminante da estatura, tanto de crianças em idade escolar quanto de menores de cinco anos $(p=0,0000)$. No estado, a proporção de retardo estatural identificada entre as crianças em idade escolar $(16,9 \%)$ foi significativamente maior quando se compara com a proporção $(12,1 \%)$ verificada entre os menores de cinco anos. Nas comparações relacionadas à distribuição espacial, constatou-se que as prevalências de déficit estatural eram estatisticamente equivalentes em menores de cinco anos e naqueles em idade escolar residentes na RMR e do IU, diferindo ambos, de forma significativa, na comparação com os resultados obtidos no IR.

Apenas entre as crianças em idade escolar, a prevalência de déficit estatural foi significativamente diferente entre o gênero masculino $(21,1 \%)$ e o feminino $(12,9 \%)$. Dentro de cada grupo etário, todas as variáveis atuaram como discriminantes estatisticamente significantes em relação ao risco de ocorrência de retardo estatural, com as prevalências aumentando na presença de situações mais adversas. Nas comparações intergrupos, as prevalências de déficit de estatura se equivaliam quando se consideravam as condições socioambientais mais satisfatórias, resultando, no entanto, em diferenciações estatisticamente significantes nas categorias com rendimentos mais desfavoráveis, condição de saneamento, tratamento da água de beber, abastecimento de água e destino do lixo. As diferenças intergrupos (idade escolar e menores de cinco anos) se mantiveram estatisticamente significativas independentemente da disponibilidade ou não de geladeira no domicílio (Tabela 1).

Na análise multivariada dos fatores de risco para o déficit estatural, com aplicação do modelo de regressão logística, foram consideradas como variáveis "candidatas" aquelas que na análise bivariada apresentaram um nível de significância de no máximo $10 \%$, para então aceitar as variáveis participantes do modelo com um valor máximo de $p=5 \%$, garantindo, dessa forma, os $95 \%$ de confiança referidos no método, o que resultou na utilização de todas as variáveis, exceto o gênero.

Vale destacar que foram ajustados modelos considerando as variáveis escolaridade e idade das mães categorizadas e contínuas e que para a escolha do melhor modelo, tal como já referido no método, ficaram as variáveis com nível de significância de, no máximo, 5\%. O modelo estatisticamente adequado definiu como variáveis explicativas:

$\mathrm{X}_{1}=$ condição nutricional do irmão em idade escolar: com déficit estatural ou sem déficit;

$\mathrm{X}_{2}=$ renda domiciliar per capita em salário mínimo, em três categorias:

1) $<1 \mathrm{SM}$ (menos de 1), 1ґ2 SM (entre 1 e menor que 2), $\geq 2$ SM (maior ou igual a 2);

2) $X_{3}=$ escolaridade materna: em anos de escolaridade;

3) $X_{4}$ = idade materna: em anos.

A Tabela 2 apresenta os valores das odds ratios resultantes da análise bivariada e da multivariada para as variáveis explicativas do déficit estatural em menores de cinco anos. $\mathrm{Na}$ análise bivariada, a renda per capita inferior a $1 / 4$ do salário mínimo foi a condição que determinou as maiores chances $(O R=6,58)$ de déficit estatural no menor de cinco anos. Já no modelo multi- 
variado, evidencia-se que o poder explicativo da renda per capita diminui e a freqüência de déficit estatural encontrado na criança em idade escolar passa a ser o fator que mais se relaciona com a baixa estatura no irmão menor de cinco anos $(O R=2,60)$.

Tabela 1. Déficit estatural em crianças, por faixa etária, segundo variáveis selecionadas. Recife, 1997

\begin{tabular}{|c|c|c|c|c|c|}
\hline \multirow[b]{2}{*}{ Variáveis } & \multicolumn{2}{|c|}{ (< de 5 anos) } & \multicolumn{2}{|c|}{ (6ト12 anos) } & \multirow[b]{2}{*}{$\begin{array}{c}\text { Estatística } \\
\left(\mathrm{Z}_{\text {calculado }}\right)\end{array}$} \\
\hline & Prevalência (\%) & $\begin{array}{l}\text { Teste } \chi^{2} \text { de } \\
\text { associação }\end{array}$ & Prevalência (\%) & $\begin{array}{l}\text { Teste } \chi^{2} \text { de } \\
\text { associação }\end{array}$ & \\
\hline \multicolumn{6}{|l|}{ Gênero } \\
\hline $\begin{array}{l}\text { Feminino } \\
\text { Masculino }\end{array}$ & $\begin{array}{l}12,3 \\
12,0\end{array}$ & $\begin{array}{l}\chi^{2}=0,02 \\
p=0,8886\end{array}$ & $\begin{array}{l}12,9 \\
21,1\end{array}$ & $\begin{array}{l}\chi^{2}=10,37 \\
p=0,0013\end{array}$ & $\begin{array}{l}-0,3223^{(\text {ns) }} \\
-4,5256^{(*)}\end{array}$ \\
\hline \multicolumn{6}{|c|}{ Abastecimento d'agua } \\
\hline $\begin{array}{l}\text { Rede geral } \\
\text { Outras formas }\end{array}$ & $\begin{array}{r}7,9 \\
17,5\end{array}$ & $\begin{array}{l}\chi^{2}=42,66 \\
p=0,0000\end{array}$ & $\begin{array}{r}9,0 \\
23,7\end{array}$ & $\begin{array}{l}\chi^{2}=33,02 \\
p=0,0000\end{array}$ & $\begin{array}{l}-0,6985^{(\mathrm{ns})} \\
-2,7498^{(*)}\end{array}$ \\
\hline \multicolumn{6}{|c|}{ Tratamento água beber } \\
\hline $\begin{array}{l}\text { Tratada } \\
\text { Sem tratamento }\end{array}$ & $\begin{array}{r}8,2 \\
17,6\end{array}$ & $\begin{array}{l}\chi^{2}=40,22 \\
p=0,0000\end{array}$ & $\begin{array}{r}9,6 \\
24,0\end{array}$ & $\begin{array}{l}\chi^{2}=31,94 \\
p=0,0000\end{array}$ & $\begin{array}{l}-0,8886^{(\mathrm{ns})} \\
-2,7642^{(*)}\end{array}$ \\
\hline \multicolumn{6}{|l|}{ Esgoto sanitário } \\
\hline $\begin{array}{l}\text { Ligado à rede } \\
\text { Fossa com tampa } \\
\text { Outras formas }\end{array}$ & $\begin{array}{c}5,8 \\
10,9 \\
17,6\end{array}$ & $\begin{array}{l}\chi_{2}^{2}=43,69 \\
p=0,0000^{(1)}\end{array}$ & $\begin{array}{r}4,6 \\
10,9 \\
26,1\end{array}$ & $\begin{array}{l}\chi_{2}^{2}=51,85 \\
p=0,0000\end{array}$ & $\begin{array}{l}0,5968^{\text {(ns) }} \\
0,0024^{\text {(ns) }} \\
-3,4655^{(*)}\end{array}$ \\
\hline \multicolumn{6}{|l|}{ Destino do lixo } \\
\hline $\begin{array}{l}\text { Coletado } \\
\text { Outras formas } \\
\text { Geladeira }\end{array}$ & $\begin{array}{r}8,2 \\
16,1\end{array}$ & $\begin{array}{l}\chi_{1}^{2}=29,79 \\
p=0,0000\end{array}$ & $\begin{array}{r}7,9 \\
23,2\end{array}$ & $\begin{array}{l}\chi^{2}=35,09 \\
p=0,0000\end{array}$ & $\begin{array}{l}-0,1661^{(\mathrm{ns})} \\
-3,3753^{(*)}\end{array}$ \\
\hline $\begin{array}{l}\text { Tem } \\
\text { Não Tem }\end{array}$ & $\begin{array}{r}7,4 \\
17,8\end{array}$ & $\begin{array}{l}\chi^{2}=50,76 \\
p=0,0000\end{array}$ & $\begin{array}{r}9,5 \\
24,1\end{array}$ & $\begin{array}{l}\chi^{2}=32,62 \\
p=0,0000\end{array}$ & $\begin{array}{l}7,1390^{(*)} \\
8,4317^{(*)}\end{array}$ \\
\hline \multicolumn{6}{|l|}{ Renda familiar } \\
\hline $\begin{array}{l}\geq 2 \mathrm{SM} \\
11-2 \mathrm{SM} \\
<1 \mathrm{SM}\end{array}$ & $\begin{array}{r}8,3 \\
15,7 \\
16,9\end{array}$ & $\begin{array}{l}\chi_{2}^{2}=28,21^{(1)} \\
p=0,0000\end{array}$ & $\begin{array}{l}11,1 \\
20,1 \\
25,3\end{array}$ & $\begin{array}{l}\chi_{2}^{2}=20,84^{(1)} \\
p=0,0000\end{array}$ & $\begin{array}{l}-1,6252^{\text {(ns) }} \\
-1,5512^{\text {(ns) }} \\
-2,3235^{(*)}\end{array}$ \\
\hline \multicolumn{6}{|l|}{ Renda per capita } \\
\hline $\begin{array}{l}\geq 0,50 \\
0,25 \vdash 0,50 \\
<0,25\end{array}$ & $\begin{array}{r}4,5 \\
14,3 \\
19,4\end{array}$ & $\begin{array}{l}\chi_{2}^{2}=76,39^{(1)} \\
p=0,0000\end{array}$ & $\begin{array}{r}3,1 \\
14,7 \\
25,4\end{array}$ & $\begin{array}{l}\chi_{2}^{2}=47,29^{(1)} \\
p=0,0000\end{array}$ & $\begin{array}{l}0,8715^{\text {(ns) }} \\
-0,1578^{\text {(ns) }} \\
-2,2456^{(*)}\end{array}$ \\
\hline
\end{tabular}

(1) Teste Qui-quadrado para tendência; (ns) Diferenças não significantes; ${ }^{(*)}$ Diferenças estatisticamente significantes.

Tabela 2. Resumo das análises para predição do déficit estatural em crianças menores de cinco anos. Recife, 1997.

\begin{tabular}{|c|c|c|c|c|}
\hline \multirow{2}{*}{ Variáveis } & \multicolumn{2}{|c|}{ Análise bivariada ${ }^{(1)}$} & \multicolumn{2}{|c|}{ Análise multivariada ${ }^{(1)}$} \\
\hline & OR & IC & OR & IC \\
\hline \multicolumn{5}{|c|}{ Condição estatural do irmão em idade escolar $\left(X_{1}\right)$} \\
\hline Sem déficit estatural & & 1 & & \\
\hline Com déficit estatural & 3,24 & $(1,79 ; 5,86)$ & 2,60 & $(1,39 ; 4,88)$ \\
\hline \multicolumn{5}{|l|}{ Renda per capita $\left(\mathbf{X}_{2}\right)^{(*)}$} \\
\hline$\geq 0,50$ & & 1 & & 1 \\
\hline $0,25 \vdash 0,50$ & 5,41 & $(2,01 ; 14,51)$ & 2,03 & $(0,89 ; 4,63)$ \\
\hline$<0,25$ & 6,58 & $(2,52 ; 17,22)$ & 2,45 & $(1,07 ; 5,64)$ \\
\hline Escolaridade materna (em anos) $\left(X_{3}\right)(2)$ & 0,84 & $(0,77 ; 0,92)$ & 0,85 & $(0,77 ; 0,94)$ \\
\hline Idade materna (em anos) $\left(X_{4}\right)(2)$ & 0,96 & $(0,93 ; 1,00)$ & 0,93 & $(0,90 ; 0,95)$ \\
\hline
\end{tabular}

${ }^{(*)}$ Teste $\chi^{2}$ para tendência; (1) Todos com $p \leq 0,10$; (2) Variáveis consideradas no modelo como contínuas. 
Tabela 3. Probabilidades estimadas de déficit estatural em crianças menores de cinco anos, segundo algumas variáveis explicativas. Recife, 1997.

\begin{tabular}{|c|c|c|c|c|c|}
\hline \multirow{2}{*}{ Renda per capita (SM) } & \multirow{2}{*}{$\begin{array}{c}\text { Condição estatural } \\
\text { do escolar }\end{array}$} & \multicolumn{2}{|c|}{ Idade da mãe analfabeta } & \multicolumn{2}{|c|}{ Idade da mãe com 11 anos de estudo } \\
\hline & & 20 anos & 35 anos & 20 anos & 35 anos \\
\hline \multirow[t]{2}{*}{$<0,25$} & Com déficit & 57,8 & 30,1 & 19,4 & 7,0 \\
\hline & Sem déficit & 34,5 & 14,2 & 8,4 & 2,8 \\
\hline \multirow[t]{2}{*}{$0,25 \vdash 0,50$} & Com déficit & 53,1 & 26,3 & 16,6 & 5,9 \\
\hline & Sem déficit & 30,3 & 12,1 & 7,0 & 2,3 \\
\hline \multirow[t]{2}{*}{$\geq 0,50$} & Com déficit & 35,8 & 15,0 & 8,9 & 3,0 \\
\hline & Sem déficit & 17,7 & 6,3 & 3,6 & 1,2 \\
\hline
\end{tabular}

As probabilidades estimadas de déficit estatural em menores de cinco anos em função das variáveis explicativas encontram-se detalhadas na Tabela 3. Em qualquer situação, a chance de uma criança menor de cinco anos apresentar déficit estatural foi, no mínimo, duas vezes maior no caso de seu irmão em idade escolar apresentar déficit de estatura. A probabilidade de déficit estatural no menor de cinco anos diminui, em qualquer situação, com o aumento da renda e foi sempre maior entre as crianças cujas mães eram mais jovens e com baixa escolaridade.

Constata-se ainda que, na melhor condição aqui evidenciada, ou seja, o irmão em idade escolar não apresentar déficit estatural, a mãe ser mais velha (35 anos de idade), ter 11 anos de escolaridade (ensino médio completo) e a família possuir renda per capita superior a 0,5 SM, o menor de cinco anos tem 1,2\% de probabilidade de ter déficit estatural. Sob exposição às condições mais precárias, quando o irmão em idade escolar apresenta baixa estatura para idade, a mãe é mais jovem (25 anos de idade), analfabeta e a renda domiciliar per capita é inferior a 0,25 SM, estima-se em $57,8 \%$ a probabilidade do menor de cinco anos ter déficit de estatura.

\section{DISCUSS Ã O}

Considerando que a altura alcançada na idade escolar reflete o efeito cumulativo de processos prévios ocorridos nos primeiros anos de vida, passou-se a recomendar o uso da medida de altura de escolares como forma de avaliar a situação de saúde e nutrição de populações no período mais vulnerável do crescimento físico. Assim, espera-se que os resultados obtidos por meio da avaliação estatural de crianças em idade escolar sejam semelhantes aos identificados entre crianças menores de cinco anos, de modo que inquéritos antropométricos de escolares possam valer como indicadores proxi da situação epidemiológica prevalente no segmento biológico mais exposto aos efeitos sinérgicos das carências nutricionais e comorbidades.

Como demonstraram os resultados, a prevalência de déficit de estatura foi significativamente maior nas crianças em idade escolar que nos menores de cinco anos no conjunto do Estado de Pernambuco (16,9\% versus 12,1\%) e no interior rural (27,1\% versus $17,4 \%)$. Já na RMR e no IU as prevalências de retardo estatural se equivaleram, o que significa que nesses dois espaços e na época do estudo o indicador altura/ idade pode ser isonômico para os dois grupos etários, enquadrando-se, portanto, nos requisitos propostos pela Organización Panamericana de la Salud (OPS) ${ }^{9}$.

Na realidade, a divergência na comparação das prevalências de déficit estatural entre crianças menores de cinco anos e em idade escolar, no conjunto de resultados obtidos para Pernambuco, se explica pela participação da amostra das famílias rurais, que representam cerca de $25 \%$ da população total do Estado. Em outras palavras, a diferenciação que ocorre no total é explicada pela participação do segmento demograficamente minoritário - o meio rural -, já que no setor urbano (região metropolitana e 
cidades do interior) a situação seria homogênea, quando considerada a prevalência.

Resultados discrepantes em relação à expectativa e, por extensão, à recomendação técnica de adotar a avaliação de escolares como proxi da situação de menores de cinco anos, também foram descritos por Carvalho ${ }^{25}$, tendo por base dados a população do Estado da Paraíba, limítrofe com Pernambuco. No entanto, em seu caso, a diferença tinha sentido contrário, sendo o déficit estatural significativamente maior em menores de cinco anos que em escolares.

Considerando o possível viés de natureza metodológica, no estudo de Carvalho ${ }^{25}$, foi estimada indiretamente pela utilização de um modelo preditivo ${ }^{26}$, enquanto a situação estatural dos escolares foi estimada diretamente em alunos ingressantes na primeira série de escolas públicas, a conclusão era a mesma: a distribuição do estado de nutrição de escolares, segundo o indicador altura/idade, não corresponderia em termos estatísticos à distribuição do estado nutricional dos menores de cinco anos, produzindo uma estimativa grosseiramente superestimada.

Ainda em relação ao estudo de Carvalho ${ }^{25}$, cabe registrar que sua interpretação, fundamentada em dados secundários, era que as escolas seriam socialmente seletivas, não refletindo a situação ou o contexto representativo das famílias dos menores de cinco anos. De qualquer maneira, o questionamento básico prevalece: uma avaliação de alunos de sete a oito anos de escolas públicas não estima, com a esperada consistência e validade, o estado de nutrição de menores de cinco anos nos mesmos espaços geográficos considerando o mesmo período do estudo.

Neste estudo, esse viés foi contornado na medida em que se efetuou a comparação entre sujeitos em idade escolar (e não escolares formalmente matriculados) e a situação de seus irmãos menores de cinco anos. Portanto, trata-se de um estudo do tipo individualizado, ao invés de uma abordagem de natureza ecológica, como na pesquisa conduzida por Carvalho ${ }^{25}$. Por que então o desencontro de resultados no Estado da Paraíba (com escolares) e em Pernambuco (com crianças em idade escolar, e menores de cinco anos), contrariando o pressuposto da OPS ? ${ }^{9}$ A conjectura mais razoável parece ser a seguinte: são contextos epidemiológicos distintos, submetidos, ainda, a etapas distintas do rápido processo de transição nutricional vivenciado no país.

Nos espaços em que a transição se encontra numa etapa mais avançada, evoluindo para uma linha de estabilidade, com a tendência de correção de déficit estatural, as condições nutricionais de crianças em idade escolar e de menores de cinco anos tendem a ser equivalentes. Ao contrário, numa etapa de rápidas mudanças, como ocorre em outros espaços do país (caso das Regiões Norte e Nordeste), com consideráveis diferenças identificadas para o meio rural27, as crianças mais velhas (em idade escolar) refletem uma situação defasada, enquanto as mais novas (caso dos menores de cinco anos) passam a expressar resultados mais favoráveis do processo de mudança. Desse modo, seriam estágios diacrônicos descritos como sincrônicos.

Os aspectos analíticos considerados na identificação estatística e na análise multivariada dos fatores de risco apontam contribuições interessantes para compreender o papel das variáveis envolvidas na gênese do comprometimento estatural. A superioridade da ocorrência de déficit estatural em crianças em idade escolar do gênero masculino $(21,1 \%$ em comparação a $12,9 \%$ no gênero feminino), não se reproduzindo em menores de cinco anos, provavelmente reflita uma característica epidemiológica do final da década de $80^{28}$ superada na segunda metade dos anos $90^{22}$. Seria, assim, uma manifestação do processo transicional em curso, sem excluir a interferência hipotética da diferenciação puberal no intervalo de 10-12 anos.

A mesma lógica poderia ser aplicada para entender o comportamento distinto de outras variáveis (abastecimento de água, tratamento da água de beber, esgotamento sanitário, destino do lixo, renda familiar), revelando-se em riscos estatisticamente significativos nas comparações 
intragrupos, mas sem efeitos quantitativamente identificados nas análises intergrupos em relação às condições ambientais e socioeconômicas mais favoráveis.

Nessas situações, estaria se completando o processo de transição e, portanto, se atenuando ou desaparecendo as diferenças de ocorrência e seus respectivos fatores de risco. Inversamente, nas condições mais adversas, o processo ainda estaria em pleno curso, com resultados diferenciais entre as crianças mais velhas (em idade escolar) e mais novas (menores de cinco anos).

Por último, uma consideração interessante: os resultados finais da análise multivariada permitiram construir "cenários de probabilidades" ilustrativos da complexa rede de causalidade do estado nutricional e de suas implicações conceituais e práticas, além dos aspectos anteriormente comentados. É relevante assinalar que na melhor combinação de fatores favoráveis (renda, condição nutricional do irmão em idade escolar, escolaridade e idade materna), o risco de déficit estatural se reduz a 1,2\%, proporção bem menor que a ocorrência registrada pelo padrão internacional de normalidade $(2,3 \%$, na tabela norte-americana do National Center Health Statistical). Não se trata de um dado aleatório, tendo em vista que resultados semelhantes foram observados, há mais de 10 anos, no Estado do Ceará, tendo por base uma amostra com 4513 observações ${ }^{29}$.

Isso significaria que o próprio padrão internacional não expressaria todo o potencial de crescimento linear da criança. Ou seja, a manifestação fenotípica da estatura de crianças norte-americanas normais não revela o potencial genotípico de crescimento, uma vez que crianças do Nordeste (Ceará e Pernambuco), em contextos seletivamente favoráveis, conseguem ultrapassar o valor limítrofe de -2 escore-Z. A tendência secular do crescimento padrão continua como um processo ainda em evolução.

Por outro lado, no pior cenário, reunindo, simultaneamente, os fatores biológicos e sociais adversos, a chance de déficit estatural se elevava para cerca de $58 \%$, simulando, assim, situações epidemiológicas que coexistem em países africanos e asiáticos ${ }^{30}$. Os resultados obtidos são semelhantes aos descritos por Sousa ${ }^{29}$, tendo por base os dados do Ceará, trabalhando com o envolvimento de análises relativas às variáveis renda, escolaridade materna, saneamento e peso ao nascer das crianças. Nas duas situações (Pernambuco e Ceará) demonstra-se o grande espectro de riscos escalonados, revelando-se a heterogeneidade de condições de riscos que não são evidenciados nas simplificações analíticas das relações bivariadas.

É possível que essas observações sejam válidas para situações mais gerais, freqüentemente encontradas no Brasil e na América Latina, de modo que, antes de aplicar, de forma acrítica, o pressuposto da analogia da situação nutricional de menores de cinco anos e escolares a partir dos resultados da estatura, é recomendável um estudo prévio de validação do indicador epidemiológico, evitando-se possíveis distorções e impropriedades envolvendo análises dos resultados.

\section{REFER Ê NCIAS}

1. Tanner JM. A History of the study of human growth. Cambridge: Cambridge Universty Press; 1981.

2. Habicht JP, Martorell R, Yarbrough C, Malina RM, Klein RE. Height and weight standards for preschool children: how relevant are ethnic differences in growth potencial? Lancet. 1974; 1:611-5.

3. Giugliani ER. Baixa estatura: um mal da sociedade brasileira. J Pediatr. 1994; 70(5):261-2.

4. Rissin A. Estado nutricional de crianças menores de cinco anos: uma análise epidemiológica no Brasil e, especialmente no Nordeste, como referência para a fundamentação de programas de intervenção nutricional. Recife, 1997 [dissertação]. Recife: Instituto Materno Infantil de Pernambuco; 1997.

5. França Júnior I, Silva GR, Monteiro CA. Tendência secular da altura na idade adulta de crianças nascidas na cidade de São Paulo entre 1950 e 1976. Rev Saúde Pública. 2000; 34(Supl 6):102-7.

6. Waterlow JC. Observations on the natural history of stunting. In: Linear growth retardationin less Developer countries. New York: Nestlé; 1988. Nutrition Workshop series no 14. 
7. Waterlow JC. Protein-energy malnutrition the nature and extint of the problem. Clin Nutr. 1997; 16(Suppl 1):3-9.

8. Monteiro CA, Torres AM. Can secular trendes in child growth be estimated from a single cross sectional survey? Br Med J. 1992; 305(6857): 797-9

9. Organización Panamericana de la Salud. Vigilancia Alimentaria y Nutricional in las Americas. Indicadores Nutricionales en los sistemas de vigilancia alimentaria y nutricional. Washington (DC); 1989. OPS. Publicación Científica n 516.

10. Carvalho AT, Costa MJC, Ferreira LOC, Batista Filho M. Retardo estatural em escolares na Paraíba: variações de gênero e idade nas quatro mesorregiões fisiográficas do Estado. Rev IMIP. 1998; 12:24-9.

11. Carvalho AT, Costa MJC, Ferreira LOC, Batista Filho M. Cartografia do retardo estatural em escolares do Estado da Paraíba, Brasil. Rev Saúde Pública. 2000; 34(1):3-8.

12. Monteiro CA. Coleta e análise da altura dos alunos ingressantes nas escolas de primeiro grau do país: uma proposta para um sistema nacional de acompanhamento do estado de saúde e nutrição da população. J Pediatr. 1989; 65:89-92.

13. Valverde $V$, Rojas $Z$, Vinocur $P$, Payne $P$, Thompson A. Organization of an information system for food and nutritional programmes in Costa Rica. Food Nutr Bull. 1981; 7(1):32-40.

14. Valverde V, Delgado $H$, Flores S, Sibrian R, Palmieri $M$. The school as a source for food and nutrition surveillance systems in Central America and Panama. Food Nutr Bull. 1985; 7:32-7.

15. Valverde V, Delgado H, Flores S, Sibrian R, Palmieri $M$. Uses and constrainsts of school children's height data for planning purposes: national experiences in Central America. Food Nutr Bull. 1986; 8:42-8.

16. Parillon CD, Valverde $V$, Delgado $H$, Newman $B$. Distribuición politico administrativa del estado nutricional segun el censo de talla de ninõs escolares del primer grado en Panama. Arch Latinoam Nutr. 1988; 38(1):42-54.

17. Mondini L, Monteiro CA, Lei DLM, Cordelini S. A coleta da altura de alunos ingressantes nas escolas de primeiro grau em um sistema de vigilância nutricional: qualidade dos dados e análise de custo em dois municípios brasileiros. J Pediatr. 1994; 70(5):267-72.

18. Guerra VMCO, Oliveira AA, Souza MER, Dantas MJBL, Sá MLB, Girão MSN. Primeiro censo estadual de altura/idade dos escolares de primeira série do ensino fundamental. Fortaleza: Secretaria Estadual de Educação; 1993.
19. Rivera F, Rivera MA, Leitão S, Carvalho A, Moura HJ, Benígna MJ, et al. A desnutrição crônica por municípios nos estudantes da primeira série do primeiro grau da sétima região educacional do Estado da Paraíba. Rev Nutr. 1994; 7(2):113-31.

20. Piauí. Secretaria de Educação e Cultura. Fundação de Amparo ao Estudante. Primeiro censo estadual de altura/idade dos escolares de primeira série do ensino fundamental do Estado do Piauí. Teresina: A Secretaria; 1993.

21. Batista Filho M \& Romani SAM, organizadores. Alimentação, nutrição e saúde no Estado de Pernambuco. Recife: Instituto Materno Infantil de Pernambuco; 2002. Série Publicações Científicas $\mathrm{n}^{\circ} 7$.

22. Laurentino GEC. Prevalência da desnutrição em crianças menores de 5 anos, do Estado de Pernambuco e sua relação com alguns fatores de risco-1997 [dissertação]. Recife: Departamento de Nutrição, Centro de Ciências da Saúde da Universidade Federal de Pernambuco; 1997.

23. World Health Organization. Use and interpretation of anthropometric indicators of nutritional status. Bull World Health Organ. 1986; 64(6):929-41.

24. Brasil. Instituto Brasileiro de Geografia e Estatística. Pesquisa Nacional por Amostra de Domicílio-PNAD [CD-room]. Rio de Janeiro: IBGE; 2002.

25. Carvalho AT. Nanismo em escolares do Estado da Paraíba: uma abordagem ecológica [dissertação]. Recife: Instituto Materno Infantil de Pernambuco; 1997.

26. Benício MHD'A, Monteiro CA. Desnutrição infantil nos municípios brasileiros: risco de ocorrência. São Paulo: NUPENS/USP; 1997.

27. Batista Filho $M$, Rissin A. A transição nutricional no Brasil: tendências regionais e temporais. Cad Saúde Pública. 2003; 19(Supl 1):S181-91.

28. Reichenheim ME, Harpham T. Perfil Intracomunitário da deficiência nutricional: estudo de crianças abaixo de cinco anos numa comunidade de baixa renda do Rio de Janeiro (Brasil). Rev Saúde Pública. 1990; 24(1):69-79.

29. Sousa FJP. Determinantes socioeconômicos da desnutrição infantil no Estado do Ceará [dissertação]. Fortaleza: Universidade Federal do Ceará; 1991.

30. Onis M, Frongillo EA, Blossner M. Is malnutrition declining? An analysis of changes in levels of child malnutrition since 1980. Bull World Health Organ. 2000; 78(10):1222-33.

Recebido em: 8/11/2004

Versão final reapresentada em: 21/6/2005 Aprovado em: 19/7/2005 